\title{
The Best Manifold Theory in the Frequency Domain of Time Dependent Functions an Application to: Seismic Engineering
}

\author{
J.L. Urrutia-Galicia \\ Instituto de Ingeniería, Mecánica Aplicada, UNAM \\ E-mail:jurg@pumas.iingen.unam.mx
}

(recibido: enero de 2004; aceptado: diciembre de 2004)

\begin{abstract}
The paper presents a novel civil engineering interpretation of the Fourier spectrum and phase of a seismic record. When the concept of linear manifolds is accounted for, we can generate clear interpretations of the maximum projections of a seismic event. Choosing the greatest ordinates of a given spectrum is equivalent to the selection of the frequency space where the maximal projections are recorded. The use of these maximum ordinates generates the original seismic record with great accuracy and with a minimum of data. This fact clearly indicates that most of the information about a time dependent signal is encoded in this best manifold. This fact is relevant, as it will clearly reduce the processing time, (depending on every record) up to $90 \%$.
\end{abstract}

Keywords: Signal analysis, Fourier analysis, best manifold solution, amplitude spectrum, phase spectrum, $\mathbf{1 / f}$ ( $\mathbf{1}$ over $\mathbf{f}$ noise) and Earth's translation.

\section{Resumen}

El artículo presenta una nueva interpretación (ingeniería civil) del espectro y fases de Fourier de un registro sísmico. Cuando se usa el concepto de espacios lineales coordenados, podemos generar interpretaciones claras de las máximas proyecciones de un evento sísmico. Escoger las máximas proyecciones de un espectro dado es equivalente a la selección del espacio de frecuencia en donde las máximas proyecciones son registradas. El uso de estas máximas ordenadas recupera un evento sísmico. Escoger las máximas proyecciones de un registro sísmico original con gran exactitud y con un mínimo de datos. Este hecho indica claramente que la mayor parte de la información de una señal dependiente del tiempo está codificada en el mejor espacio. Esto último es muy relevante, ya que reduce el tiempo de proceso, (dependiendo de cada registro) hasta en un $90 \%$.

Descriptores: Análisis de señales, análisis de Fourier, mejor espacio de solución espectro de amplitud, espectro de fase, $\mathbf{1 / f}$ ( 1 sobre $\mathbf{f}$ ruido) y translación de la Tierra.

\section{Introduction}

The transmission of signals for different engineering fields is of paramount importance in present times. During the early 1960s and 1970s, when computers were very expensive, the applications included areas such as mechanical (Tse et al., 1978) and civil engineering (Clough and Penzien, 1975), where the main issue is apparently concerned with the spectrum of a signal regardless of the phase. However, other more sophisticated applications (Steven, 1977a) are found in areas such as: telecommunications, transferring information from one location to another, audio processing, where most of the digital signal processing is related to image and audio transmission, echo location, in radar and sonar devices, mainly for military applications and in image processing, not only for video entertainment but also for medical use such as computed tomography scanning. In this context, the key 
The Best Manifold Theory in the Frequency Domain of Time Dependent Functions ...

areas are (Steven, 1977a): "Radar \& Sonar, where national security (in different developed countries) is involved; oil exploration, where large amounts of money could be made; space exploration, where the data are irreplaceable; and in medical imaging, where lives could be saved. Since these four areas involved either national security or industrial property, the research material was turned into classified material (Steven, 1977b).

Some areas of scientific knowledge have been left behind, using a theoretical background that was already abandoned in the more developed areas of knowledge such as electronics, radar and sonar engineering. With the advent of personal computers in the 80s and 90s the Digital Signal processing technology was now promoted by the incredible market posed by cellular technology, the computer revolution and all the range of applications such as image compression and transmission, fax, etc. When the author (Urrutia, 1984) first encountered "the best manifold problem in the field of stability of circular cylindrical shells" it was clear that given a particular $\mathrm{n}^{\text {th }}$ space or manifold, the maximum projections of a function $F(x)$ or $F(t)$ on different coordinate functions ${ }_{n}(x)$ or ${ }_{n}(t)$ could fall in different coordinates not necessarily gathered in the same "corner space". This, however, is not that evident, as in the case of some accelerograms where, the presence of these maximum projections are regarded as traces of the original record mixed with some kind of noise. In fact during posterior analyses these maximum are eliminated, smoothing the original record through some averaging process that involves low, medium and high frequency amplitudes of the absolute value of the Fourier amplitude spectrum. The phase is explicitly ignored and regarded as useless (Tse et al ., 1978).

In view of these facts, the objective of this paper will be to adapt some of the main ideas of (Urrutia, 1984) the "Best manifold concept of Shell Theory" and those of the more advanced technology (Steven, 1977b) of "Digital Signal Processing" in order to apply these results to the digital processing of seismic engineering records.

As a side result it will be seen that the controversial problem of the curiosity called (Steven, 1977c) 1/f noise (one over f noise) present in very diverse systems, at low frequencies below $10 \mathrm{~Hz}$, can and will be explained in due course.

\section{Theory}

Given a certain domain of length $\mathrm{T}$ and a linear space generated by an infinite set of coordinate functions ${ }_{n}(t)$, then any function $f(t)$ can be represented by the following equation:

$$
f(t) \quad a_{n}(t)
$$

Among the many choices of coordinate functions, two of the first and most well known are the harmonics $\sin (t)$ and the $\cos (t)$ functions. In this sense, any arbitrary function in general is neither symmetric nor asymmetric at the same time and it must be possible to generate such a function as the linear combination of the projections $a_{n}$ and $b_{n}$ of the function $f(t)$ as follows:

$$
f(t) a_{n 0} a_{n} \cos \frac{n t}{T} \quad b_{n} \sin \frac{n t}{T}
$$

Given a particular function $f(t)$ we are usually interested in functions or signals with a time duration $0 \quad t \mathrm{~T}$; therefore, it is the same if we use either one of the two manifolds $\cos (t)$ or $\sin (t)$. For the sake of simplicity we choose the linear space generated by the coordinate functions $\sin (t)$ reducing equation (1) to the more simple way

$$
f(t) \quad b_{n=1} \sin \frac{n t}{T}
$$

Note that every function $\sin (n \pi t / T)$ in the present work is explicitly regarded as a coordinate function (a coordinate) in such a way that the function $f(t)$ will be decomposed, like a common vector by means of its projections $a_{n}$ times the coordinate $\widetilde{x}_{n}$ that in the present case stands for $\sin (n \pi t / T)$.

Following the nomenclature presented by William (1972) the function ${ }_{n}=\sin (n \pi t / T)$ is rewritten as follows $n=\sin (n \Delta \omega t)$ where $=\mathrm{T}$. In this way the excitation frequency of every $n$ harmonic is $n=n \Delta$. It is clear (Steven, 1977c) that $a_{n}$ (the projection of any arbitrary function $f(t)$ into the coordinate function $n=\sin (n \pi t / T))$ and $n$ at the excitation frequency $n=n \Delta$ completely defines the harmonic contribution of every periodic wave form. If we plot the value of the 
projection $a_{n}$ versus the excitation frequency ${ }_{n}=$ $n \Delta$, the result is a series of continuous vertical lines at frequencies $n \Delta$ for $n=1,2, \ldots$, with $=/ T$, this is shown in figure 1 (a).

However, another usual way to plot the coefficients $a_{n}$ is the one shown in (Steven, 1977c), figure 1 (b) as $\left|a_{n}\right|$ that is called the Fourier Spectrum (with all the values $a_{n}$ positive) of the arbitrary function $f(t)$. The results of figures 1 (a) and (b) are represented by continuous fine lines in figures 2 (a) and (b). These last two curves or functions by themselves are not the same; the smoothing process is usually performed on these ones. The smoothing of the thin curve of figure 1 (a) will lead to a thick line that tends to cross the horizontal axis the same number of times that the thin line does and the thick line of figure 2 (b) will never cross the horizontal $n$ axis just as the thin line does. This last fact is commonly observed in current literature when we see the smoothing of some accelerogram via some averaging process.
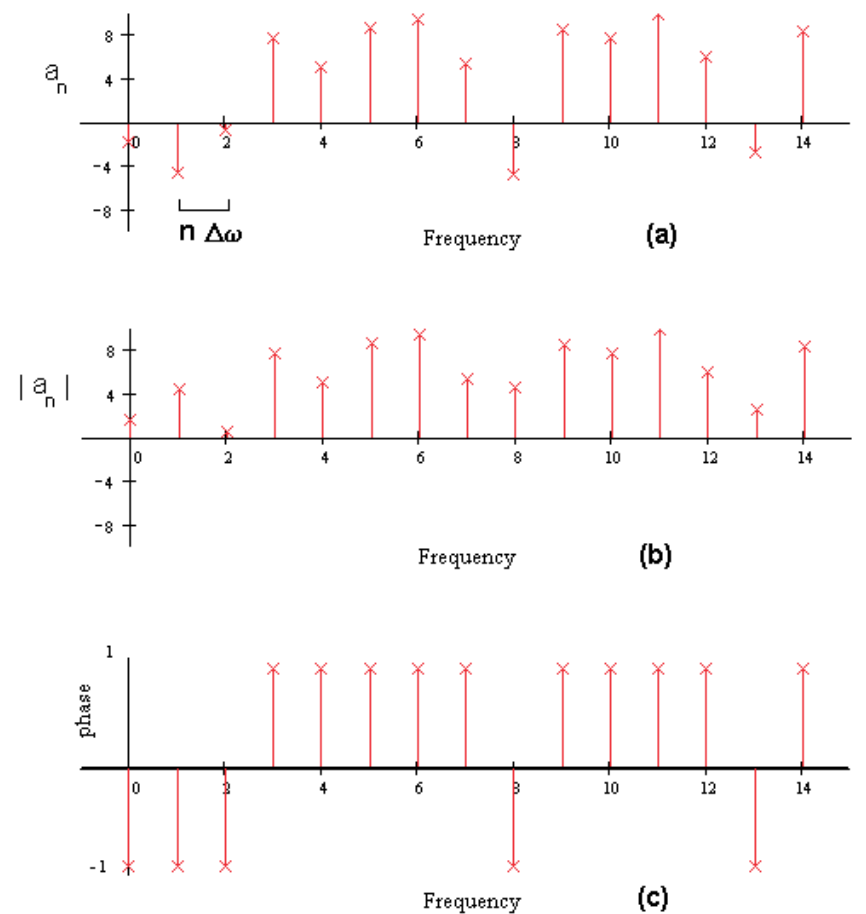

Figure 1
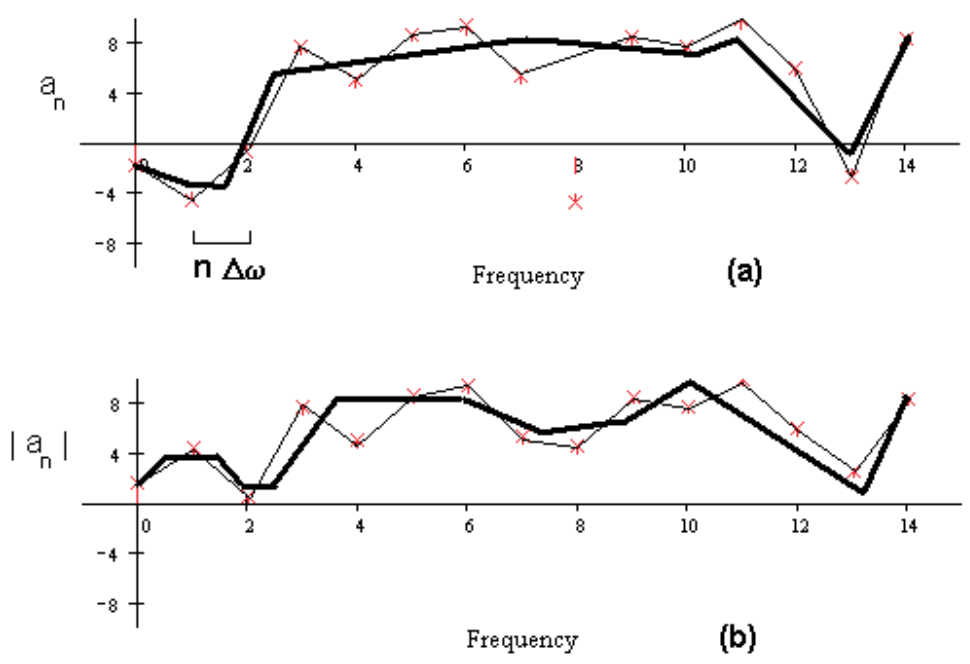

Figure 2 
The Best Manifold Theory in the Frequency Domain of Time Dependent Functions ...

The conclusion from this is that the thick curve of figure 2 (a) is a much better approximation to the real frequency recorded signal (the thin line), than the (always non negative) thick signal that approaches the conventional Fourier Spectrum (thin line) of figure 2 (b). The reconstruction of the original $f(t)$ will be much more accurate if we use the $a_{n}$ values of the smoothed thick curve of figure 2 (a) than the reconstruction done with the $a_{n}$ coefficients of the thick curve of figure 2 (b).

As we will see the problem of accuracy just reviewed can be eliminated if the original Fourier Spectrum (with all $a_{n}$ being positive) is preserved along with its phase that is encoded in the one plus (1) or one minus ( -1 ) versus frequency record shown in figure 1 (c). This will be clearly shown with examples and under the light shed by the findings of reference (Urrutia, 2002) for the analysis of base motion problems.

\section{Fourier Analysis}

The scheme analysis to calculate the Fourier coefficients $A_{m}$ and $B_{m}$ was developed elsewhere, and we shall not dwell on this. If more information is needed in regard to this algorithm reference (Urrutia, 1982) is recommended. Nevertheless, we mention that the main feature of this algorithm is that given a finite " $n$ " number of data points the algorithm will retrieve as many coefficients as needed (many times more than " $n$ "), that means more accuracy in comparison with the standard algorithms that will only provide " $n / 2$ " coefficients. The $B_{n}$ coefficients are obtained from the following formula

$$
\cos m_{a} \frac{2 k 1}{2} c \quad \cos m_{a} \frac{\mathrm{W}_{k}}{m} \frac{2 k \quad 3}{2} c
$$

for completeness we note that $m$ stands for the $m$ coefficient associated with the $\sin (m \pi t / T)$ function (where $t$ is the time variable), $\mathrm{W}_{k}$ stands for the $k$ amplitude of accelerogram taken every $t$ seconds (increment of time), $a=\mathrm{t} / \mathrm{T}$ stands for the frequency increment in the algorithm, $\mathrm{T}$ is the signal or earthquake's duration and " $c$ " is a constant that allows sifting of the original function, on the $[0,2]$ domain. As mentioned earlier, the whole algorithm and theory can be found in reference (Urrutia, 1982).

\section{Signal's Modelling}

In the general theory of Fourier analysis it is common to represent an arbitrary time dependent function $f(t)$ written as follows

$$
f(t) \quad B_{m} \sin \frac{m t}{T}
$$

However, in the Theory of Shells (Steven, 1977b) it is seen that if the solution has maximum projections on different space regions then it is better to write our approximation $f(t)^{*}$ as follows

$$
\begin{gathered}
f(t)^{*} \text { Factor }{ }^{{ }_{m}^{m 2} B_{m} \cos \frac{m t}{T}}{ }_{m m 3}^{m 4} B_{m} \sin \frac{m t}{T} \\
{ }_{m b 5}^{m 6} B_{m} \cos \frac{m t}{T} \text { etc }
\end{gathered}
$$

where $m 1$ and $m 2, m 3$ and $m 4$ and $m 5$ and $m 6$ are the points in the frequency domain that enclose the maximum ordinates of the calculated spectrum and the term Factor is a correction made in order to match the maximum positive ordinate of the original record reproduced by equation (5) with $m$ running from 1 to . We shall see this with example records obtained in Mexico City during different earthquakes.

\section{Accelerogram Recorded at the SCT (Ministry Of Communications And Transportation) In Mexico City}

A particularly interesting accelerogram was recorded on the early morning of September $19^{\text {th }}$ of 1985. The EW component is shown in figure 3 and the corresponding Fourier spectrum (frequency -vs- coefficients $\left|B_{n}\right|$ amplitude) is indicated in figure 4. Besides these two figures we also show in figure 5 the Fourier spectrum with the $\mathrm{B}_{m}$ (with its proper sign) coefficients -vs- the frequency of the coordinate $\sin (m \pi t / T)$. In order to see the convergence of our approximations we 
now prefer to draw figures 4 and 5 in the amplitude (Steven, 1977a); (William, 1972), $\mathrm{B}_{m}-\mathrm{vs}-$ the frequency number $m$ of the coordinate $\sin (m \pi t / T)$ as it is seen in figures 6 and 7 .

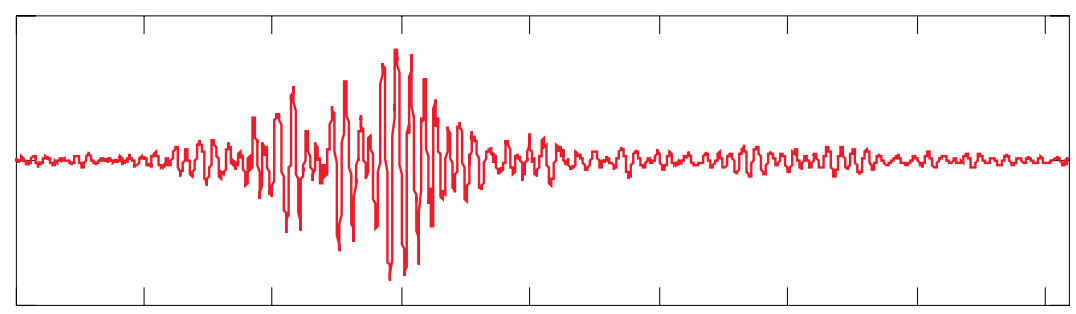

Figure 3

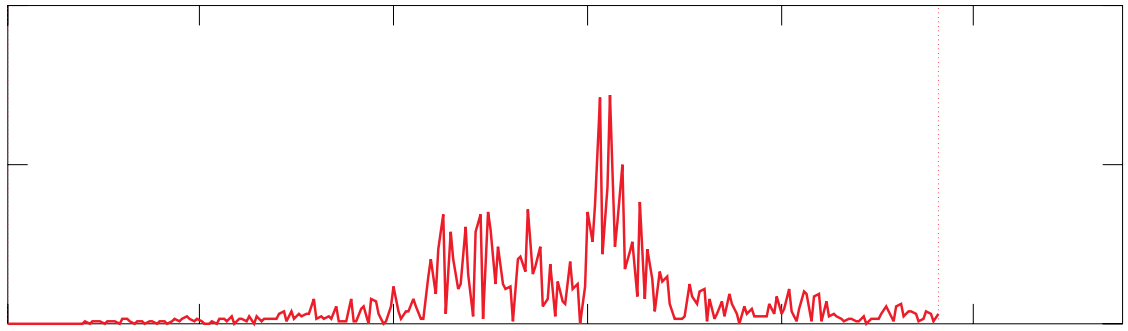

Figure 4

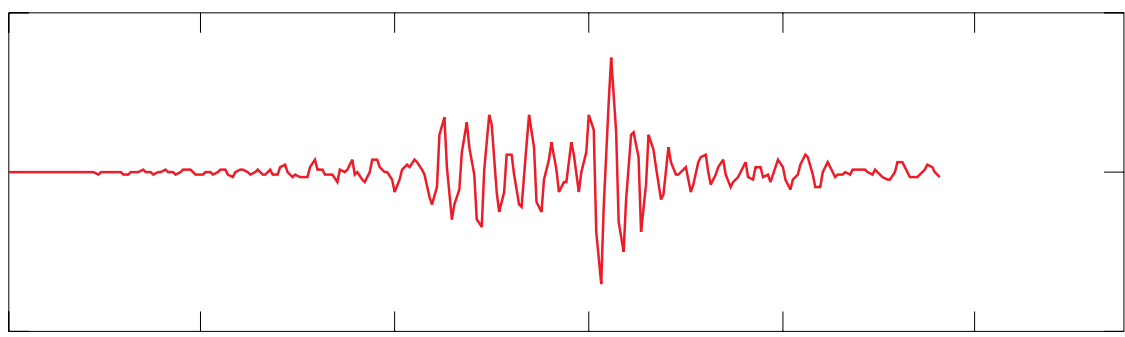

Figure 5

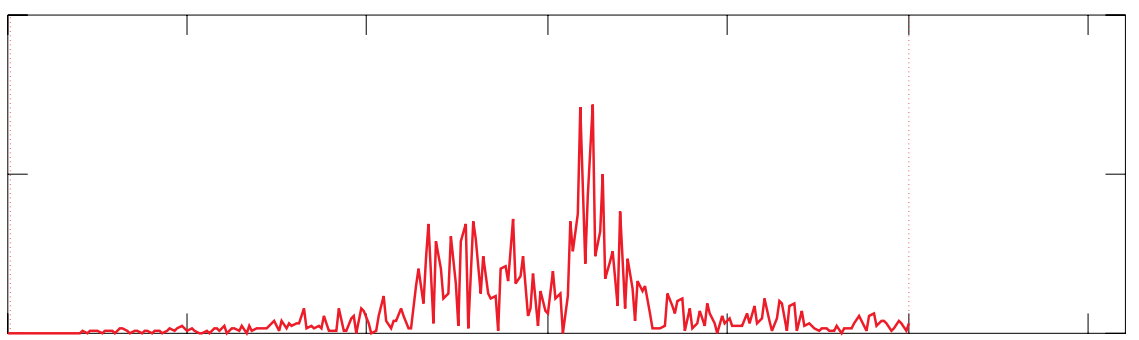

Figure 6. $\left|\mathrm{B}_{m}\right|$ coefficients, classical spectrum 
The Best Manifold Theory in the Frequency Domain of Time Dependent Functions ...

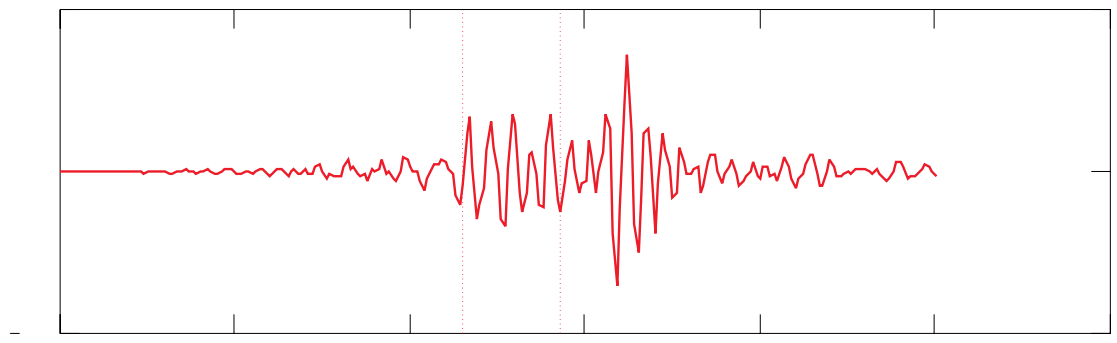

Figure 7. $\mathrm{B}_{m}$ coefficients

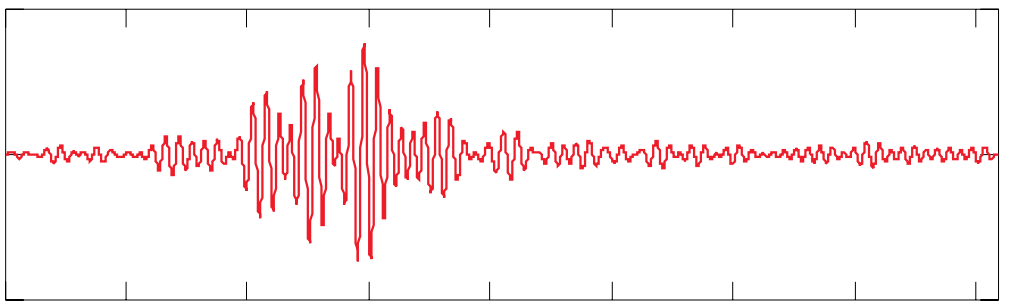

Figure 8

The advantage of figure 7 in the wave number domain, over that over the frequency domain shown in figure 5 , is the space notion dealing with coordinates ${ }_{m}=\sin (m \pi t / \mathrm{T})$ and their corresponding wave numbers $m$ and with the ordinates (the $\mathrm{B}_{m}$ amplitudes) of any arbitrary function $f(t)$ on the coordinates $m$. This concept of projections becomes crucial in getting back an approximation $f(t)^{*}$ of the original $f(t)$ from a local space with maximum ordinates as the following one:

$$
f(t)^{*} \text { Factor }{ }_{m m 1}^{m 2} B_{m} \cos \frac{m t}{T}
$$

where the term Factor stands for a correction factor, normally just a little bit greater than one, that compensates for the loss energy, neglecting the rest of the zone $m<m 1$ and $m>m 2$. In general the approximation $f(t)^{*}$ can have one or more maximal as it is indicated in equation (6).

For an approximate reproduction figure 8 , of the original signal we use the following approximation

$$
f(t) * \text { Factor }{ }_{m m 1}^{m 2} B_{m} \cos \frac{m t}{T}{ }_{m m 3}^{m 4} B_{m} \sin \frac{m t}{T}
$$

with $m 1=115$ and $m 2=143$ as it is indicated in figure 7 and from $m 3=155$ to $m 4=170$ where the next region of maximum ordinates is located. The result, seen in figure 8 , includes a Factor $=1.054$ in order to match the approximation $f(t) *$ to the function values of the accelerogram of figure 3 . At first sight the two accelerograms of figures 3 and 8 are identical and to achieve this only a few harmonics of "the best manifold" are needed. We used only 19 harmonics from 115 to 143 plus 16 harmonics more from 155 to 170 for a total of 35 harmonics. Notice that the SCT record used in the analysis has 4087 points. This fact means that should we use the Fast Fourier Transform scheme we would need $2^{12}=4096$ points to comply with the requirements of the FFT computer code. Then it is well known that the total number of coefficients that the FFT algorithm will lead to is 2048 and in the present work we calculated only 250 sine related coefficients and from those only 35 were significant in reproducing the original SCT record of the September 19 of 1985 earthquake. It is clear that considering the ordinates (the Fourier coefficients or projections) of the spectrum of figure 7 rather than that of figure 6 is a more suitable approach than that of considering the spectrum of figure 6 alone. If two envelopes were drawn, one touching the maximum positive 
ordinates and another one covering the maximum negative ordinates of figure 3 , the envelopes drawn for the approximation of figure 8 would be identical and an anew observation would be in the order. In referenceit (Steven, 1977d) is established that much (I would rather say all) of the information about the shape of the time domain waveform is contained in the phase, rather that in the magnitude. To this we now add, "Most of the information about the shape of the time domain waveform is encoded in the best manifold and in the phase, rather that in the magnitude". This last statement completes the panorama and will be illustrated in the following examples. Notice, that this is not always true as in the case of audio signals where the information is mostly encoded in the magnitude, with the phase playing a minor role, reference (Steven, 1977a) explains the reasons that sustain this statement. In the former example, we used equation (8) with the coefficients $B_{n}$ obtained from the spectrum of figure 6 with the original sign or the coefficients obtained directly from figure 7 . In the following step we now shift the plots of figures 6 and 7, 50 places to the right to obtain figures 9 and 10. The 250 coefficients are now plotted from the wave number 50 to 300 as it is indicated from Al to A2 in both figures.

The maximum ordinates of figures 6 and 7 are shifted from $m=162$ to 212 . To generate the corresponding signals in time domain we replace equation (8) by the following expression

$$
\begin{gathered}
f(t) * \text { Factor } \\
{ }_{m m 1}^{m 2} B_{m} \cos \frac{[m+n] t}{T}{ }_{m=3}^{m 4} B_{m} \sin \frac{[m+n] t}{T}
\end{gathered}
$$

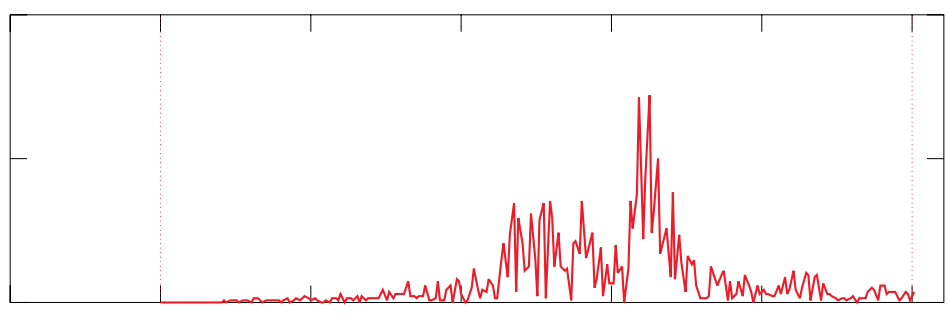

Figure 9. $\left|\mathrm{B}_{m}\right|$ coefficients, classical spectrum

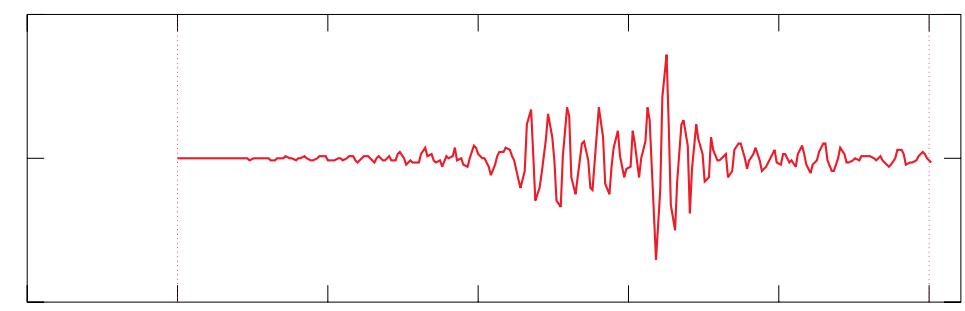

Figure $10 . \mathrm{B}_{m}$ coefficients

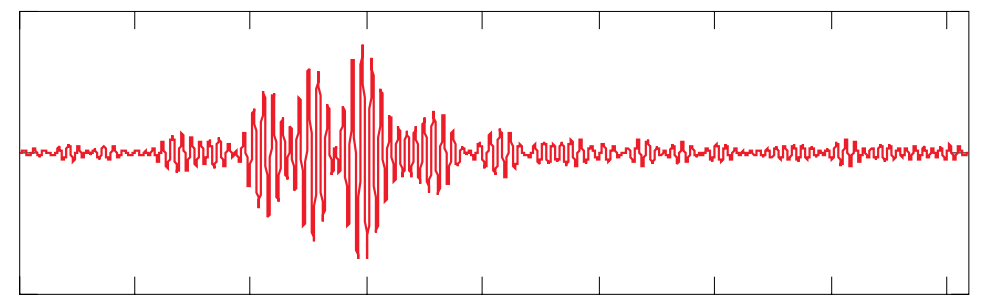

Figure 11 
The Best Manifold Theory in the Frequency Domain of Time Dependent Functions ...

If we take again the best manifold with $m 1=115$ and $m 2=143$ and $m 3=155$ to $m 4=170$ with a frequency shifting $n=50$ and a Factor $=1.0525$ the result is observed in figure 11 .

Using equation (9) again with the same parameters but using $n=-60$ will lead to figure 12 .

The plotting of $\left|\mathrm{B}_{m}\right|$ and $\mathrm{B}_{m}$ of figures 9 and 10 are shifted 50 harmonics (transforming the original record into a high frequency record) to the right but are identical in shape to the plots of figures 6 and 7 of the original record. When in equation (9) we use $n=-60$ the corresponding plots (or spectra) of $\left|B_{m}\right|$ and $\mathrm{B}_{m}$ will be shifted 60 places to the left (transforming the original record into a low frequency record) of the original SCT spectrum. We now arrive at the following major conclusion. "The acceleration records of figures 8,11 and 12 with identical (although shifted) $\left|\mathrm{B}_{m}\right|$ and $\mathrm{B}_{m}$ plots have identical envelopes covering the maximum positive and minimum negative ordinates. The frequency of figures 8,11 and 12 depends on where the spectrum $\left(\left|\mathrm{B}_{m}\right|\right.$ or $\left.\mathrm{B}_{m}\right)$ is located in the frequency domain".

\section{Accelerogram at Imp on March $4^{\text {th }}$ 1992, Surface Ground Motion, Horizontal Component}

As a second and perhaps more striking example we present a surface accelerogram, figure 13 recorded on March 4th 1992 in the Mexican Institute of Petroleum (IMP), in Mexico city.

Along with this record we present the corresponding plots of $\left|\mathrm{B}_{m}\right|$ (the classical spectrum) and $\mathrm{B}_{m}$, in figures 14 and 15 . In figure 14 four maximum ordinates are detected and the first two are indicated by ind 1 for $m=\underline{60}$ and ind2 for $\mathrm{m}=\underline{87}$. The other two maximum are located for $\mathrm{m}=\underline{111}$ and $\mathrm{m}=\underline{141}$.

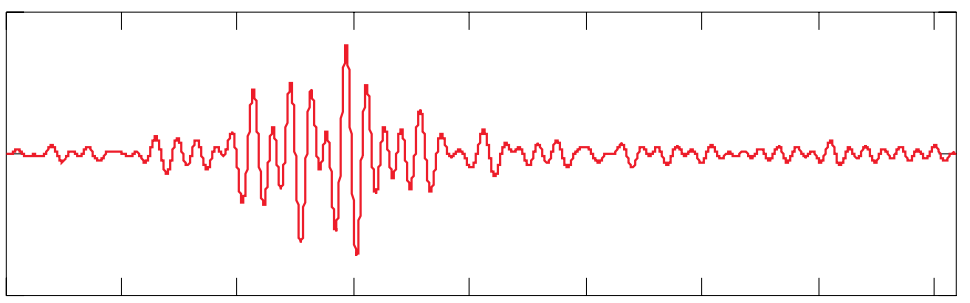

Figure 12

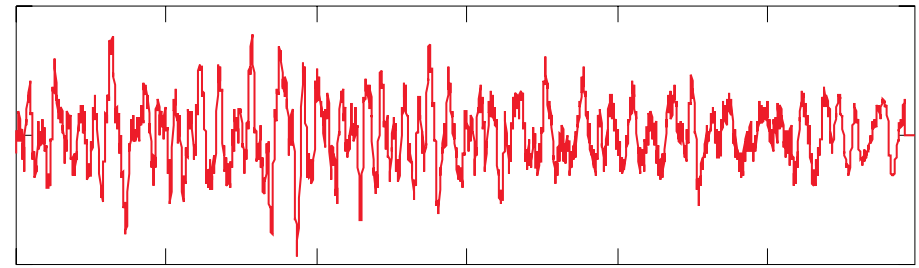

Figure 13. Accelerogram at the IMP, Mexico City

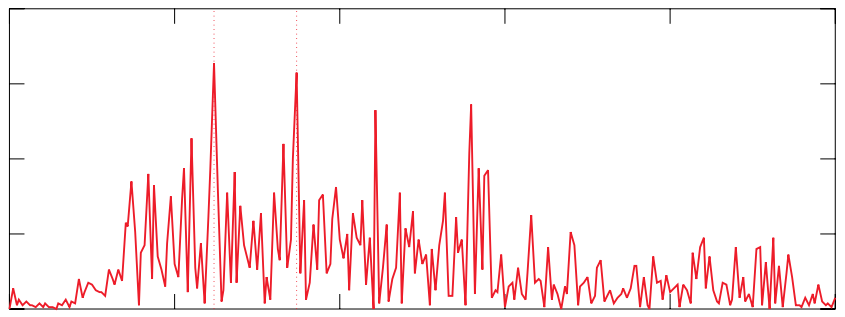

Figure 14. $\left|\mathrm{B}_{m}\right|$ coefficients. IMP classical spectrum 


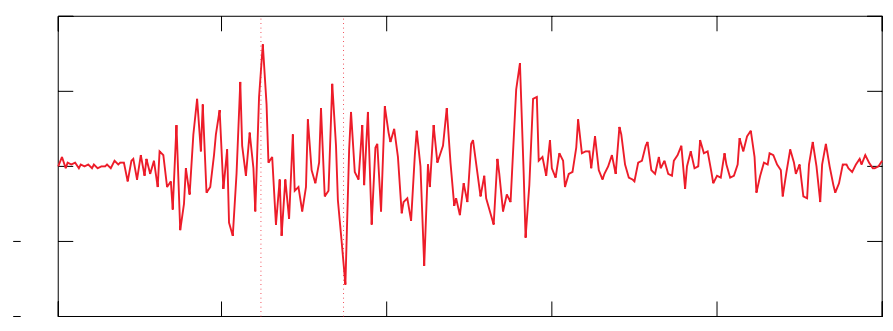

Figure 15. $\left|\mathrm{B}_{m}\right|$ coefficients. IMP record

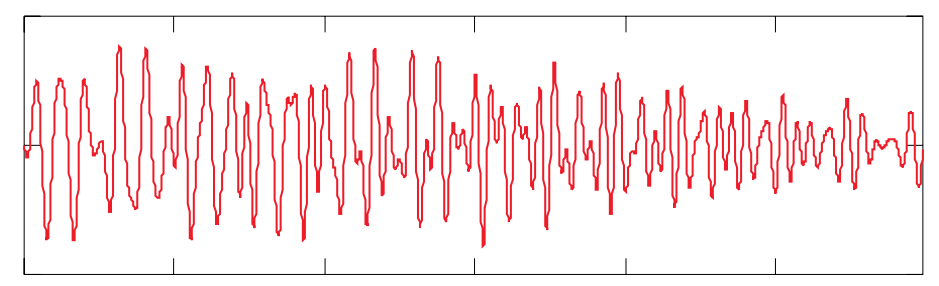

Figure 16. For IMP record

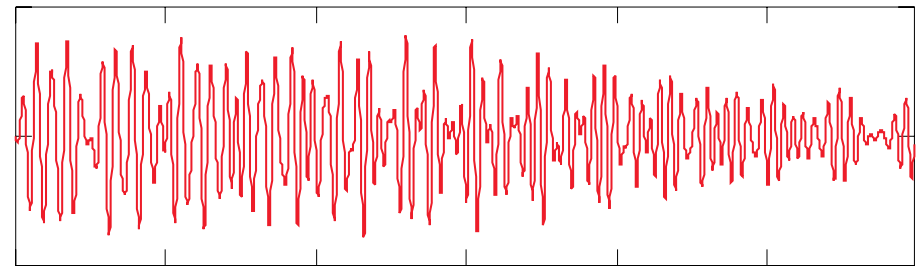

Figure 17. Spectrum shifted 50 harmonics to the right

We now rewrite equation (9) with the four best regions selected around $m=60,87,111$ and 141 as follows

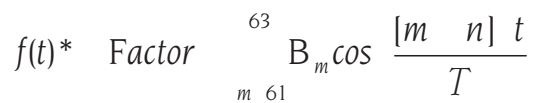

$$
\begin{aligned}
& { }_{m 86}^{88} \mathrm{~B}_{m} \sin \frac{\left[\begin{array}{ll}
m & n
\end{array}\right] t}{T}{ }_{m 110}^{112} \mathrm{~B}_{m} \sin \frac{[m n] t}{T} \\
& { }_{m 140}^{142} \mathrm{~B}_{m} \sin \frac{[m n] t}{T}
\end{aligned}
$$

If we now set Factor $=2.56$ and $n=0$ we reconstruct this signal as indicated in equation (10) and we get figure 16 with a very accurate approximation to the original record of figure 13 .

If in equation (10) we now set $\mathrm{n}=50$ the result is now shown in figure 17.

We arrive at the same conclusions as in the former example with the SCT (1985) record but now with four best manifolds (with three harmonics each). We see again that the envelope of the maximum positive and the maximum negative ordinates of figures 16 and 17 are the same. The envelopes of figure 16 contain a low frequency signal ( $\mathrm{n}=0$ in equation 10 ) and the envelopes in figure 17 contain a higher frequency signal ( $n=50$ in equation 10). The point where the 
The Best Manifold Theory in the Frequency Domain of Time Dependent Functions ...

present signal was recorded also recorded a vertical component with a very peculiar feature that shows the trace of something that had remained elusive so far.

\section{Accelerogram At Imp on March $4^{\text {th }}$ 1992, Surface Ground Motion, Vertical Component}

A very interesting case arises at this point. In signal analysis theory (Steven, 1977e), a curiosity called 1/f noise (one-over -f noise) has been measured in very diverse systems, such as traffic density on freeways and electronic noise in transistors. In spite of its wide occurrence in many areas of technology, a general theory and understanding of $1 / \mathrm{f}$ noise has not been found so far. However, in a recent work (Urrutia, 2002), by the author of this paper, on the theory of base motion analysis of structures a clue regarding this problem was found.

We take from reference (Urrutia, 2002) the corresponding displacements $\mathrm{X}_{0}, \mathrm{X}_{1}, \mathrm{X}_{2}$, and $\mathrm{X}_{3}$ of a four degrees of freedom system, these results are shown in figure $18(\mathrm{a})$ to $(\mathrm{d})$.

The absolute displacements of the corresponding four masses are those due to the

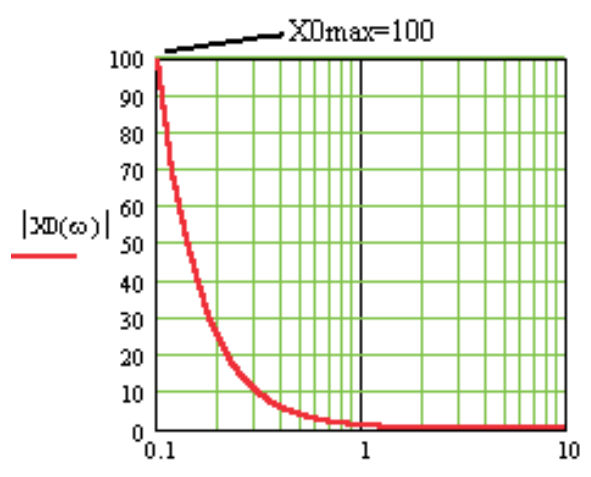

(a)

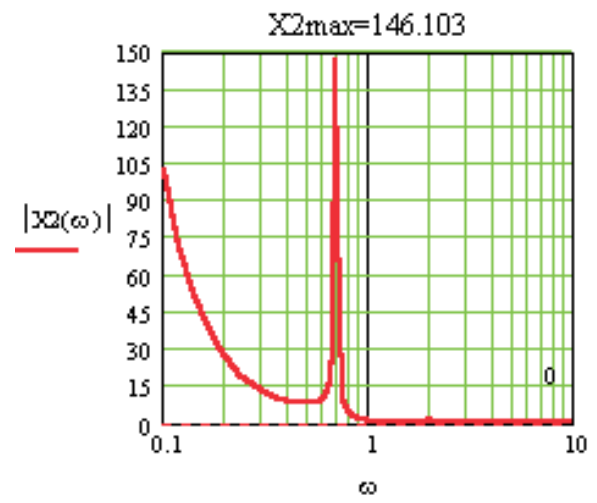

application of the huge force F0 (that produces a unit acceleration) applied to a huge supporting mass $m 0$ with a size much greater than that of the sum $m 1+m 2+m 3$ of the other three masses. The displacements now include, at frequencies lower than approximately $0.4 \mathrm{rads} / \mathrm{s}$, a rigid body component that keeps the four masses traveling in the same sense or in phase. These large responses at low frequencies (due to the rigid body component) explain the appearance in many real records of the so-called $1 / \mathrm{f}$ noise. In the case of the accelerograms that the civil engineer deals with, this rigid body component is always present and it is regarded as a base line problem and it is removed by some averaging methods that render accelerograms as those observed in figures 6 and 14 , with $B_{m}$ components, at low frequencies (of the spectrum) of negligible size. As we will see in the next example the real problem remained elusive.

Following these explanations we now present in figure 19, the vertical component of the accelerogram at IMP recorded on March $4^{\text {th }}, 1992$ (surface ground motion).

In figures 20 and 21 we show the plots of $B_{m}$ and those of $\left|B_{m}\right|$ (the classical spectrum).
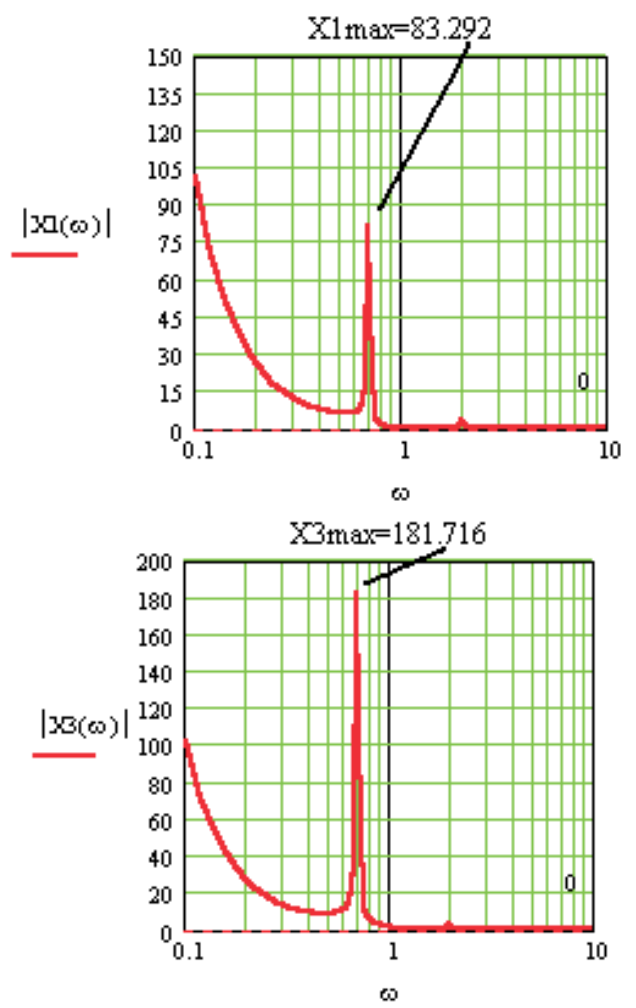

Figure 18. From (Urrutia, 2002) 


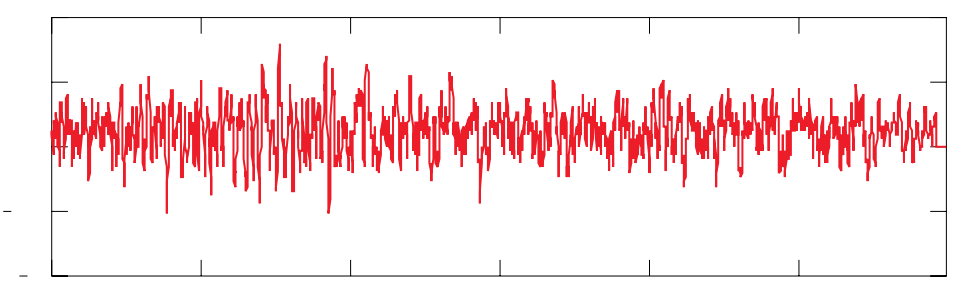

Figure 19

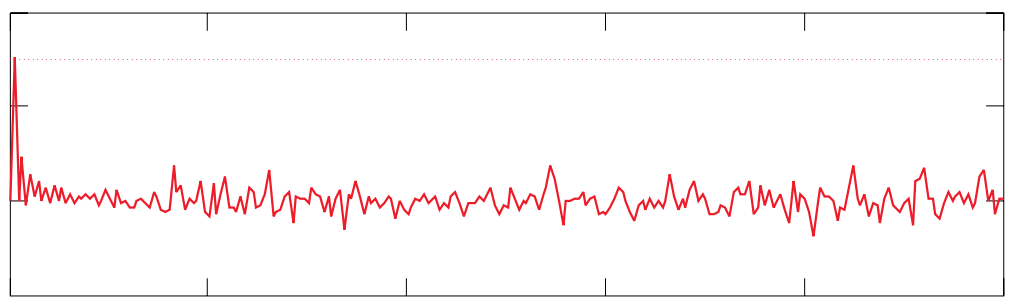

Figure 20. $\mathrm{B}_{m}$ coefficients with the proper sign

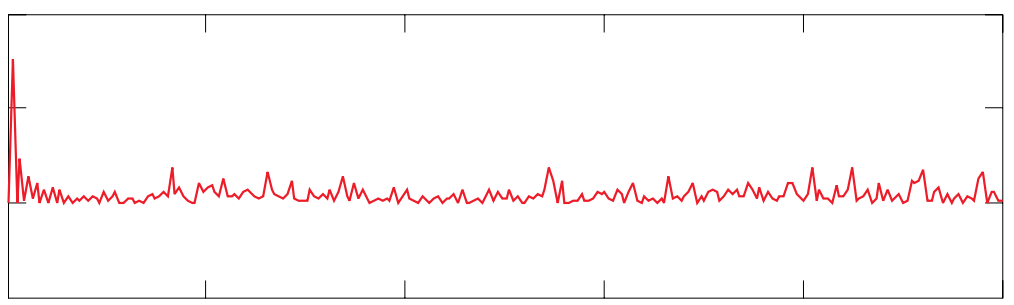

Figure 21. Classical spectrum, always positive

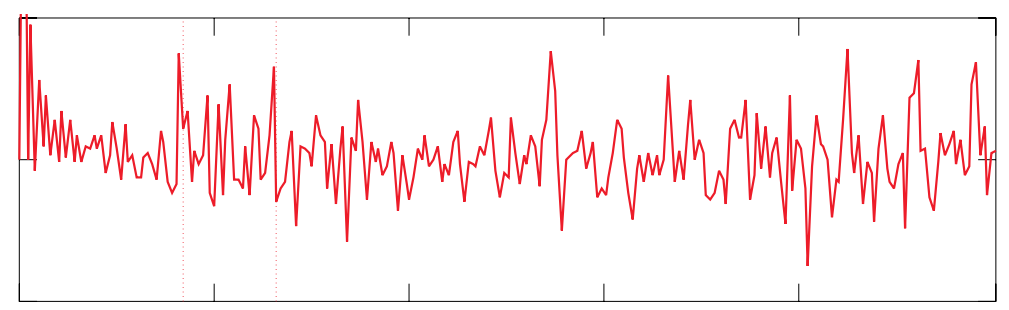

Figure 22

No matter that this record was corrected for base line, the rigid body component remains at low frequency numbers. The reason of the presence of this large component at low frequency is that the motion recorded by the accelerogram, includes a great low frequency component of the measuring point that includes the translation of the earth around the sun. Then if we are interested in the relative motion caused by an earthquake, we should simply remove the low frequency components of the accelerogram and work with the rest of the signal. A close up of figure 20 is presented in figure 22, that shows that the first two maximum are at ind $1=41$ and at ind $2=66$. 
The Best Manifold Theory in the Frequency Domain of Time Dependent Functions ...

A third maximum that we include is at $m=137$. For $\mathrm{n}=0$ and $\mathrm{a}$ Factor $=3.94$ the following approximation is possible with the three best regions selected.

$$
\begin{gathered}
f(t)^{*} \text { Factor }{ }_{m 35}^{45} \mathrm{~B}_{m} \cos \frac{[m \quad n] t}{T} \\
{ }_{m 50}^{75} \mathrm{~B}_{m} \sin \frac{\left[\begin{array}{ll}
m & n
\end{array}\right] t}{T}{ }_{m 133}^{141} \mathrm{~B}_{m} \sin \frac{[m \quad n] t}{T}
\end{gathered}
$$

The result of this approximation is seen in figure 23.

With the same equation (11) and with $n=70$ and the same Factor $=3.94$ the next result of figure 24 is attained.

The envelopes of the maximum positive and the minimum negatives of figures 23 and 24 are the same.

To end this last example we can say that, the relative motion experienced by a structure due to the earthquake of figure 19, can adequately and accurately be predicted with the simulated accelerogram of figure 23 . The $1 / \mathrm{f}$ noise problem now attributed to the long long earth's period for translation around the sun (and finally trough the galaxy) is simply deleted. The maximum information about the shape and effects of a particular earthquake in encoded in both a) the Best manifold and b) in the signs of the $B_{m}$ coefficients (the phase), more than one manifold may be chosen.

The motion due to the low frequency range is calculated with the following formula with Factor $=1$.

$$
f(t)^{*} \text { Factor }{ }_{m m 1}^{m 2} \mathrm{~B}_{n} \cos \frac{m t}{T}
$$

From $m 1=1$ to $m 2=35$ we get figure 25 .

And for $m 1=1$ to $m 2=15$ we get figure 26 .

In the last two figures the motion reaches a finite traslation and everything returns to the zero equilibrium position.

Figure 23

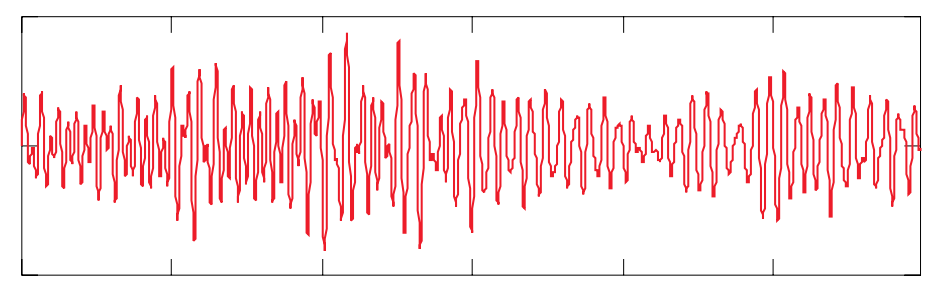

Figure 24. Shifted in frequency 


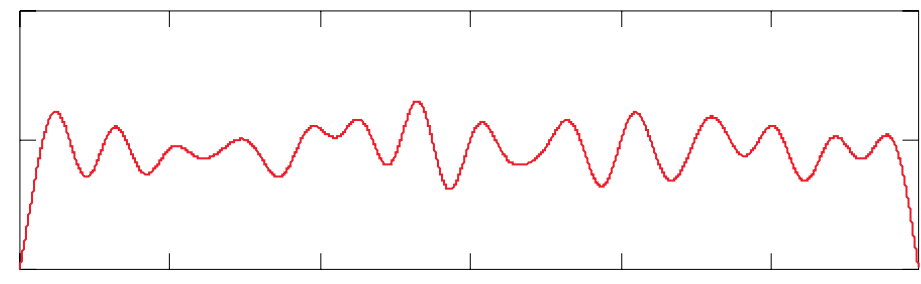

Figure 25

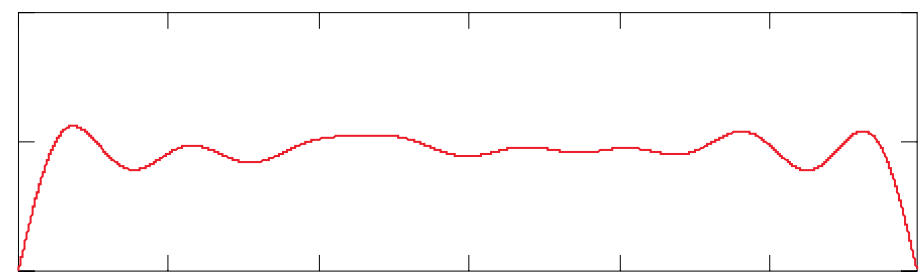

Figure 26

\section{Conclusions}

From the results presented we conclude that the most important information from the accelerograms, for dynamic analysis of structures, is encoded in the best manifold (or manifolds) and in its phase (the proper signs of the $B_{m}$ coefficients). Accurate reproductions of the original accelerogram can be achieved with the best manifold and a proper Factor chosen in such a way the amplitudes of the approximation $f(t)^{*}$ match those of the original record. It may happen that, especially in ground motions of hard soil, more than one best manifold may be needed.

Regarding the low frequency $\mathrm{B}_{\mathrm{m}}$ ordinates of the spectrum we have shown that the $1 /$ f noise problem is nothing more that the trace left behind, in the accelerograms recorded, of the long period motion of the point where the measurement is made, of the earth around the sun and finally a trace of the motion of the point of measurement trough the outer space. With respect to this last statement all that we need to do is to neglect the low frequency record and keep the rest of the record at higher frequencies. The points from where the accelerograms are important may vary from problem to problem as the circumstances dictate.

\section{References}

Clough R. and Penzien J. (1975). Dynamics of Structures. McGraw-Hill.

Steven W.S. (1977a). The Scientist and Engineer's Guide to Digital Signal Processing. California Technical Publishing, ISBN 0-9660176-3-3.

Steven W.S. (1977b). The Scientist and Engineer's Guide to Digital Signal Processing. California Technical Publishing, ISBN.

0-9660176-3-3, Chapter 3, p. 66, lines 30 to 34.

Steven W.S. (1977c). The Scientist and Engineer's Guide to Digital Signal Processing. California Technical Publishing, ISBN 0-9660176-3-3, Chapter 9, p. 172, lines 23 to 32.

Steven W.S. (1977d). The Scientist and Engineer's Guide to Digital Signal Processing. California Technical Publishing, ISBN 0-9660176-3-3, Chapter 10, p. 192, figure 10-6.

Steven W.S. (1977e). The Scientist and Engineer's Guide to Digital Signal Processing. California Technical Publishing, ISBN.

0-9660176-3-3, Chapter 10, p. 172, line 23.

Tse F.S., Morse I.E. and Hinkle R.T. (1978). Mechanical Vibrations, Theory and Applications. Ed. Allyn and Bacon Inc., Boston, Massachusetts, 2a. ed. 
The Best Manifold Theory in the Frequency Domain of Time Dependent Functions ...

Urrutia-Galicia J.L. and Sherbourne A.N. (1982). Analysis of Stresses in Internally Loaded Cylindrical Shells, Computers and Structures, Vol. 15, No. 3, p.p. 225-240.

Urrutia-Galicia J.L. (2002). Contributions to Applied Mathematics in Engineering, Berichte aus dem Konstruktiven Ingenieurbau, Universität der Bundeswehr München (GERMANY), ISSN 1431-5122, 01/2. Paper
"Base Motion Analysis of Structures, the General Semidefinite Model.

Urrutia-Galicia J.L. (1984). Stability of Fluid Filled Circular Cylindrical Shells. Ph. D. Thesis presented to the University of Waterloo, CANADA.

William T.T. (1972). Theory of Vibration with Applications. Prentice-Hall, Inc., Englewood Cliffs, N.J.

\section{Semblanza del autor}

José Luis Urrutia-Galicia. Obtuvo el grado de ingeniero civil en la Facultad de Ingeniería, UNAM en 1975; asimismo, los grados de maestría (1979) y doctorado (1984) en la Universidad de Waterloo, en Ontario, Canadá. Es investigador del Instituto de Ingeniería, UNAM en la Coordinación de Mecánica Aplicada. Sus áreas de interés cubren: matemáticas aplicadas y mecánica teórica, análisis tensorial, estabilidad y vibraciones de sistemas discretos, vigas, placas y cascarones. Ha recibido reconocimientos como el "Premio al Mejor Artículo" de las Transacciones Canadienses de Ingeniería Mecánica (CSME) (Montreal, Canadá 1987) por el artículo "The Stability of Fluid Filled, Circular Cylindrical Pipes, part II Experimental", también le fue otorgada la "Medalla Duggan", que es la más alta distinción de la CSME (en la Universidad de Toronto, Canadá, 1990) por el artículo "On the Natural Frequencies of Thin Simply Supported Cylindrical Shells. 